\title{
The entire organization of transcription units on the Bacillus subtilis
} genome

\author{
Hirokazu Kobayashi, Joe Akitomi, Nobuyuki Fujii, Kazuo Kobayashi, \\ Md Altaf-Ul-Amin, Ken Kurokawa, Naotake Ogasawara and \\ Shigehiko Kanaya*
}

Address: Department of Bioinformatics and Genomes, Graduate School of Information Sciences, Nara Institute of Science and Technology, 89165, Takayama, Ikoma, Nara 630-0192, Japan

Email: Hirokazu Kobayashi - h-kobaya@bs.naist.jp; Joe Akitomi - akitomi-jou@mxf.nes.nec.co.jp; Nobuyuki Fujii - fujii@gp.hitachi.co.jp; Kazuo Kobayashi - kazuok@bs.naist.jp; Md Altaf-Ul-Amin - amin-m@is.naist.jp; Ken Kurokawa - ken@is.naist.jp; Naotake Ogasawara - nogasawa@bs.naist.jp; Shigehiko Kanaya* - skanaya@gtc.naist.jp

* Corresponding author

Published: 28 June 2007

BMC Genomics 2007, 8:197 doi:10.1186/147|-2164-8-197
Received: 22 January 2007

Accepted: 28 June 2007

This article is available from: http://www.biomedcentral.com/I47I-2/64/8/I97

(C) 2007 Kobayashi et al; licensee BioMed Central Ltd.

This is an Open Access article distributed under the terms of the Creative Commons Attribution License (http://creativecommons.org/licenses/by/2.0), which permits unrestricted use, distribution, and reproduction in any medium, provided the original work is properly cited.

\begin{abstract}
Background: In the post-genomic era, comprehension of cellular processes and systems requires global and non-targeted approaches to handle vast amounts of biological information.

Results: The present study predicts transcription units (TUs) in Bacillus subtilis, based on an integrated approach involving DNA sequence and transcriptome analyses. First, co-expressed gene clusters are predicted by calculating the Pearson correlation coefficients of adjacent genes for all the genes in a series that are transcribed in the same direction with no intervening gene transcribed in the opposite direction. Transcription factor (TF) binding sites are then predicted by detecting statistically significant TF binding sequences on the genome using a position weight matrix. This matrix is a convenient way to identify sites that are more highly conserved than others in the entire genome because any sequence that differs from a consensus sequence has a lower score. We identify genes regulated by each of the TFs by comparing gene expression between wild-type and TF mutants using a one-sided test. By applying the integrated approach to II $\sigma$ factors and $17 \mathrm{TFs}$ of $B$. subtilis, we are able to identify fewer candidates for genes regulated by the TFs than were identified using any single approach, and also detect the known TUs efficiently.

Conclusion: This integrated approach is, therefore, an efficient tool for narrowing searches for candidate genes regulated by TFs, identifying TUs, and estimating roles of the $\sigma$ factors and TFs in cellular processes and functions of genes composing the TUs.
\end{abstract}

\section{Background}

Recent progress in genome projects has generated a vast amount of nucleotide sequence data, and analyses of gene expression by global approaches have started to broaden our understanding of cell systems. As a useful model for systems biology and genomics, many studies use Bacillus subtilis, a spore-forming gram-positive bacterium whose genome sequence has been determined [1]. The ultimate goal of post-genome analysis is to specify transcriptional regulation in the entire genome. Computational algo- 
rithms to locate transcription units (TUs) have been developed based on analysis of signal sequences that are located at the boundaries of TUs from promoters to terminators, homologous gene pairs on other genomes, intergenic distance, functional categories, and gene clusters conserved among various species [2-7]. In the present study, a string of one or more genes co-transcribed is defined as a TU [4].

Identification of transcription factors (TFs) and their binding sites on their target genes is an important element of transcriptome analysis in the post-genome-sequencing era. Until now, various approaches have been taken to identify specific DNA-binding sites of TFs. DNA-binding specificities have traditionally been determined by experimental techniques such as DNase I footprinting and electromobility shift assay $[8,9]$. More recently, putative TF binding sites have been identified by computational techniques such as hidden Markov models (HMMs) [10] and position-weight matrices (PWMs) $[11,12]$. The PWM has one column for each position in the binding site and one row for each nucleotide. Each of the matrix elements is proportional to the relative frequency of the corresponding nucleotide at each position, and the score for a particular site is the sum of the matrix values for the sequence. Therefore, PWM is often used to predict nucleotide-protein binding sites and is used in the TRANSFAC database, which covers many known TFs and binding sites [13]. This approach is a convenient way to identify positions that are more highly conserved than others in a whole genome, because any sequence that differs from a consensus sequence has a lower score. The accuracy of detecting promoter sequences thus depends on the conservation of TF-binding sites.

We can now use complete genomic DNA sequences from several species and analyze massive amount of data on differential gene expression in microarray experiments [14]. Using microarrays in various conditions, we can obtain co-expression patterns for adjacent genes, which is an important property for determining transcription units.

In the present study, we identify the TUs in B. subtilis using a combination of (i) a bioinformatics approach, using PWM methods that identify TF-binding sites by detecting statistically significant TF-binding sequences on the genome; and (ii) two DNA microarray analyses, one to predict co-expressed gene clusters by calculating Pearson correlation coefficients of expression profiles for neighboring genes, and the other to determine genes regulated by each of the TFs in the units by comparing gene expression between wild-type and TF deletion mutants in the genome.

\section{Results}

\section{The integrated strategy for TU prediction}

The procedures for elucidating TUs are outlined in Fig. 1. First, co-expressed gene clusters were determined by correlating expression profiles between neighboring genes transcribed in the same direction with no intervening gene transcribed in the opposite direction (Fig. 1(1)). Coexpression between neighboring genes was estimated using a t-test of the Pearson correlation coefficient. To predict co-expressed gene clusters on the B. subtilis genome, we used 98 DNA microarray data sets in 13 different timeseries growth conditions. We then detected various sizes of co-expressed gene clusters and observed that most clusters consisted of four genes or less.

Second, we regarded genes having promoters predicted by PWM as the start of the TUs (Fig. 1(2)). There are at least 18 different $\sigma$ factors that direct RNA polymerase, and a large number of sequence-specific DNA binding proteins that play various roles of controlling gene expression, as promoter activators or repressors in B. subtilis $[15,16]$. We then examined the TF-binding promoter sequences of 11 $\sigma$ factors except $\sigma \mathrm{A}$, which are known to possess multiple cis elements, and 17 TFs within $300 \mathrm{bp}$ upstream of an open reading frame for all 4,219 genes of $B$. subtilis by $\mathrm{PWM}$, and found putative promoters regulated by each TF below the thresholds. Figure 2A shows a comparison of coverage (Fig. 2A1) and sensitivity (Fig. 2A2) between the $1 \%$ and $5 \%$ thresholds. We were able to narrow down the candidates for TF-binding sites to $26.1 \%$ of the candidates (i.e. from 431 to 110 sites) when we changed the threshold from 5\% to $1 \%$ (Fig. 2A1). On the 5\% threshold, we identified an average of $78 \%$ known promoters, and an average of $69 \%$ promoters on the threshold of $1 \%$ (Fig. 2A2). Thus, the average difference of detecting known promoters is $9 \%$, corresponding to 3.5 promoters, by changing the threshold from $5 \%$ to $1 \%$. Furthermore, in most TFs we could efficiently narrow down candidates for the TF binding site, and found that the number of known promoter sites detected below each of the thresholds hardly changed. Therefore, we took these PWM analyses at the threshold of $1 \%$.

Third, we derived significant expression change data from TF deletion mutant microarray data to identify genes regulated by each of the TFs (Fig. 1(3)). In these analyses, we applied a one-sided test to examine genes whose expression changed significantly in the normalized microarray data, and found candidate up-regulated genes for $28 \mathrm{TFs}$, including $11 \sigma$ factors and candidate down-regulated genes for 17 TFs. Furthermore, we used the false discovery rate (FDR) procedure to remove false-positive data from the candidates of significant expression change data and narrow the candidates for genes regulated by each of the TFs [17]. 


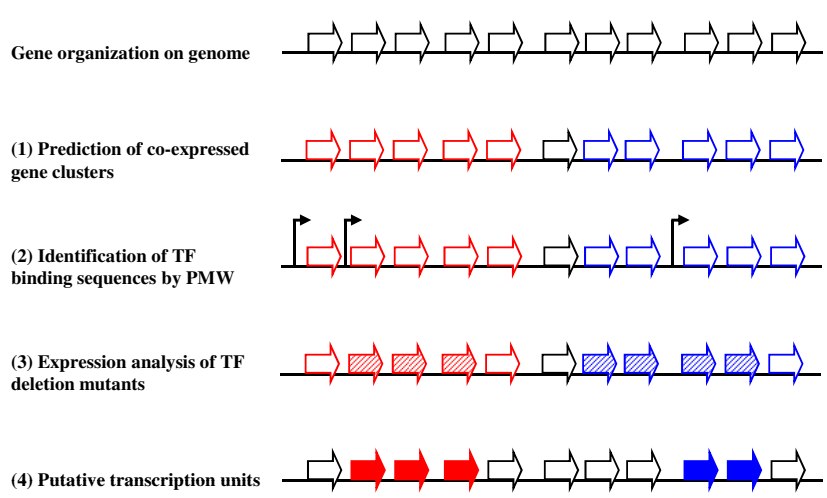

Figure I

Outline of procedure for elucidating TUs by integrated analyses. (I) Co-expressed gene clusters predicted by correlating expression profiles between neighboring genes; (2) promoter (thin arrows) prediction by PWM as the start of a TU in the putative co-expressed gene clusters; (3) detection of significantly expressed genes (stripe thick arrows) by comparison between TF deletion mutant arrays and wild ones; (4) identification of genes composing putative TUs (filled thick arrows) by integration of the three analyses.

We then integrated these analyses, and present a comparison of coverage (Fig. 2B1) and sensitivity (Fig. 2B2) between two integrated conditions (i.e. a 5\% threshold at PWM and a 5\% threshold in the deleted mutant array without FDR, and a 1\% threshold at PWM and a 5\% threshold in the deleted mutant array with FDR). We were able to narrow down the candidate genes composing TUs at the 5\% PWM without FDR to $24.5 \%$ of the candidates (i.e. from 194 to 50 genes) when we changed the condition from the PWM 5\% threshold without FDR to the PWM 1\% threshold with FDR, with $87.1 \%$ of genes detected at 5\% PWM without FDR also being detected at $1 \%$ PWM with FDR. Thus, the candidates can be effectively narrowed without remarkable loss of regulationknown genes under the condition of 1\% PWM with FDR. The $\sigma \mathrm{L}$, PerR, and PurR TUs were efficiently detected. Regarding the $\sigma \mathrm{L}$ TUs in particular, we could narrow down the 63 TU candidates for the PWM 5\% threshold to 9 candidates for the PWM 1\% threshold with FDR control without any loss of sensitivity. The detected TUs for the $1 \%$ PWM with FDR and known TUs regulated by each of the TFs are listed in Additional file 1.

\section{Organization of TUs in B. subtilis}

The difference between TUs predicted in the present study and known operons in B. subtilis indicates that most of the predicted TUs are consistent with those reported (Fig. 3). Consequently, the entire TU map on B. subtilis genome can be estimated on the basis of the predicted TUs. At the
1\% PWM with FDR, we can pick 2,183 genes composing 892 TUs, which include known operons, from the complete $B$. subtilis genome. The average size of the polycistronic transcription unit is 3.71 genes, which is comparable in size to those in Staphylococcus aureus (3.47 genes) [5] and in E. coli K12 (3.41 genes) [4]. Distribution of the TUs to the number of genes is almost identical between B. subtilis and S. aureus (Additional file 2) [5]. Thus the operon organization of those two gram-positive bacteria are fundamentally identical and are approximated by power-law equations, where the correlation of the double logarithm linear relation between the numbers of genes and of TUs composed by the genes is -0.98 in $S$. aureus and -0.97 in B. subtilis.

\section{Discussion}

In the present study, we identified various sizes of TUs regulated by each TF and detected gene clusters consisting of part of well-known operons (yabPQ regulated by $\sigma \mathrm{E}$ and divIC-yabR regulated by $\sigma \mathrm{X}$ in yabMNOPQ-divIC-yabR operon, nasDEF regulated by GInR in nasBCDEF operon, yjmEFGHIJ regulated by $\sigma \mathrm{E}$ in yjmABCDEFGHIJ operon, spoVE-murG regulated by $\sigma \mathrm{E}$ in murE-mraY-murD-spoVEmurG-murB-divIB- $y l x W X-s b p$ operon, $x y n B$ regulated by $\mathrm{XylR}$ in $y n a J-x y n B$ operon and yoxB-yoaA regulated by $\sigma \mathrm{B}$ in yoxCB-yoaA operon mentioned in Additional file 1). They are known to be regulated by internal promoters and to constitute functional components [18], for instance, yabPQ regulated by $\sigma \mathrm{E}$ that plays an important role in synthesis of the spore cortex and coat [19], and divIC-yabR regulated by $\sigma \mathrm{X}$ which is essential for the initiation of vegetative septum formation [20,21] in yabMNOPQ-divICyabR operon. Therefore, these gene clusters separated by internal promoters tend to be functional units.

Using the TU data, we examined the transcriptional regulation of genes by $11 \sigma$ factors whose promoter sequences have been characterized. The properties of individual $\sigma$ factors are as follows: five $\sigma$ factors $(\sigma \mathrm{E}, \sigma \mathrm{F}, \sigma \mathrm{G}, \sigma \mathrm{H}, \sigma \mathrm{K})$ regulate sporulation through morphological stages that involve the conversion of the growing cell to a two-cell sporangium, which ultimately proceeds to a single spore; $\sigma \mathrm{B}$ mediates the general stress response, and more than 150 protein-coding genes for general stress belong to the $\sigma B$ regulon $[22] ; \sigma L$ mediates cold-shock adaptation and regulation of the acetoin catabolic pathway [23]; $\sigma \mathrm{D}$ regulates flagellar synthesis, motility, and chemotaxis [24]; $\sigma \mathrm{M}$ mediates salt resistance [25]; and $\sigma \mathrm{X}$ and $\sigma \mathrm{W}$ play modulatory roles in extracytoplasmic function [26]. All the regulative relations of the $11 \sigma$ factors and 17 TFs to targeted genes are listed in Table S1, making it possible to characterize individual $\sigma$ factors according to the genes they target. Therefore, we classified genes belonging to each of the TUs into 19 COG (clusters of orthologous groups of proteins) functional categories [27] for estimat- 

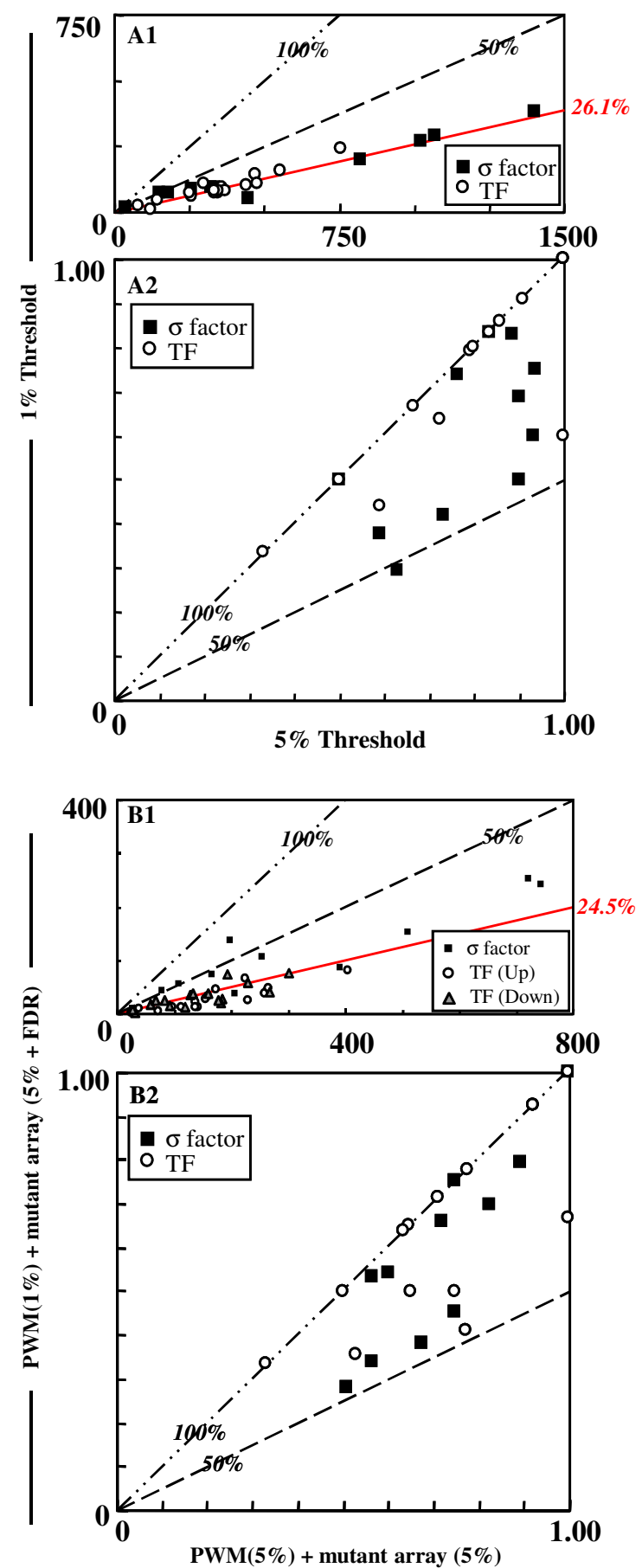

\section{Figure 2}

Comparison of coverage and sensitivity in each of the TFs. The coverage of promoters on the $B$. subtilis genome (AI), and the sensitivity of known promoter detection by PWM below the $1 \%$ threshold vs. that below $5 \%$ threshold (A2). The coverage of genes composing TUs on the $B$. subtilis genome $(\mathrm{BI})$, and the sensitivity of detection of genes composing known TUs by this integrated analysis below the 5\% threshold for PWM and the deletion mutant array without FDR vs. that below the I\% threshold for PWM and the deletion mutant array with FDR (B2). 


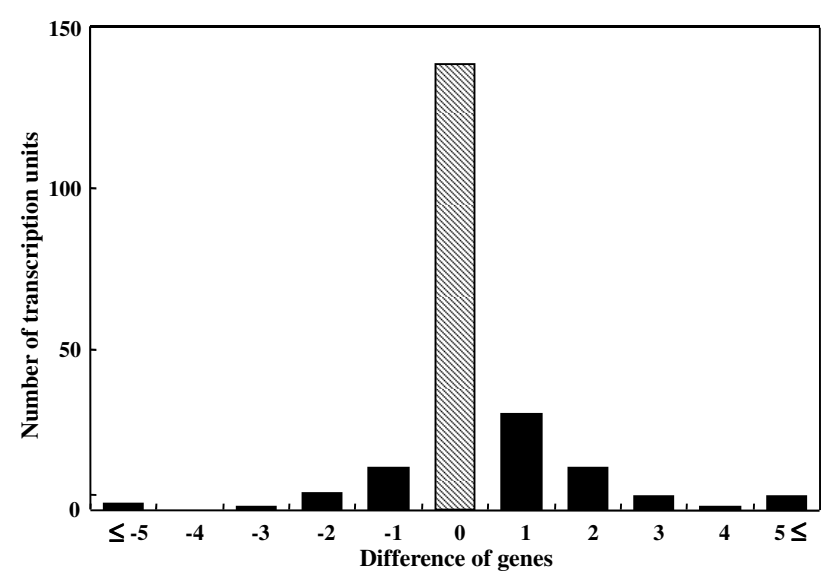

Figure 3

Comparing TUs predicted in the present study with known $B$. subtilis operons, which start with the same promoters as the TUs. $65.6 \%$ of the known TUs matched the predicted units (i.e., I 39 predicted TUs in the 212 known B. subtilis operons); when we included TUs lacking or gaining one gene of known TUs, this figure rose to $85.8 \%$. The $x$-axis indicates difference in gene number between predicted transcriptions units and known $B$. subtilis operons, and the $y$-axis indicates the number of predicted TUs.

ing the general roles of the $\sigma$ factors and TFs in cellular processes (Fig. 4).

The similarity of the roles in cellular process between individual TFs was estimated using Pearson correlation coefficients for the number of genes belonging to each of the COG categories (Fig. 4). The five $\sigma$ factors associated with regulation of the sporulation process can be classified into three groups corresponding to the sporulation process Stage 0 -III $(\sigma \mathrm{H}, \sigma \mathrm{F}$ and $\sigma \mathrm{E})$ characterized by category [J; translation, ribosomal structure and biogenesis], Stage IV $(\sigma \mathrm{G})$ characterized by the category [G; carbohydrate transport and metabolism], and Stage $\mathrm{V}(\sigma \mathrm{K})$ characterized by category $[\mathrm{M}$; cell envelope biogenesis and outer membrane]. Gene expression under the $\sigma \mathrm{G}$ control occurs in the prespore, and the main functions are to protect the spore from several hazardous conditions, high osmotic pressure [28], UV radiation and dry heat [29], and to prepare the spore for germination and outgrowth [30]. In this process, $\sigma \mathrm{G}$ regulates carbohydrate content in the cell, for example, by activating expression of the glucose dehydrogenase operon [31], controlling metabolism of the tricarboxylic acid cycle [32] and glucose uptake [33]. $\sigma \mathrm{K}$ is synthesized and becomes active in the mother cell, and directs formation of the spore coat and spore maturation [30]. Therefore, these previous experimental studies are consistent with the present results. Moreover, we can observe that each TF in a cluster has one of the frequently detected functional categories (Fig. 4). The AraR protein is well known as a negative regulator of the L-arabinose metabolic operon [34], and most of the genes negatively regulated by AraR belong to [G] (Fig. 4). Almost all the genes up-regulated by SinR are in category [N], which consists of proteins controlling cell motility and secretion, while the down-regulated genes belong to category $[\mathrm{M}]$, which consists of proteins operating cell-wall and membrane biogenesis (Fig. 4). ComK synthesis is regulated by a series of reactions that involve quorum sensing; SinR is one of the activators in this cascade, acting negatively on rok transcription [35], and is known to be a potent repressor of biofilm formation [36]. Thus, the analysis presented here agrees well with previous experimental data and enables us to assess the roles of the $\sigma$ factors and TFs in cellular processes.

In addition, the genes targeted by $\sigma$ factors and TFs are classified into 36 categories based on functional classification of the B. subtilis protein-encoding genes [37] to examine the role similarities among them based on B. subtilisspecific gene functions such as the endospore-formation process. We then show the projection of $\sigma$ factors and TFs in the largest two principal components (Fig. 5A) and factor loadings of individual categories, indicating the contribution of the category frequencies to the two principal components based on the frequencies of the 36 categories (Fig. 5B). We observe a small cluster composed of $\sigma \mathrm{D}$, CtsR and SinR (a broken line circle in Fig. 5A), which is consistent with the result in Fig. 4. Here, $\sigma D$ is the $\sigma 28$ form subunit of RNA polymerase, and many $\sigma \mathrm{D}$-dependent genes are known to be necessary for flagellar synthesis and motility functions [38]. In addition, CtsR controls the expression of heat-shock proteins that are required for stress tolerance and growth at high temperature [39], and play essential roles in competence development and motility [40]; SinR also regulates the development of genetic competence and motility [41]. Thus, the roles of these three TFs in cellular processes are associated with motility, and those are plotted in the same region of the cluster characterized by category [1.6, motility and chemotaxis] (Fig. 5A and 5B). This result shows that roles of TFs can be estimated by the principal component analysis (PCA) based on comprehensive searches for functions of gene composing these TUs.

It can also be seen in another cluster composed of Fur, Zur, IolR, PurR, RocR, and GlnR (a broken line circle in Fig. 5A). Fur and Zur regulate the expression of ABC transporters and both TFs control iron and zinc uptake and homeostasis pathways in response to available metals $[42,43]$. IolR and PurR also control transport systems. IolR regulates genes encoding inositol transporters and inositol uptake [44], while PurR regulates purine trans- 


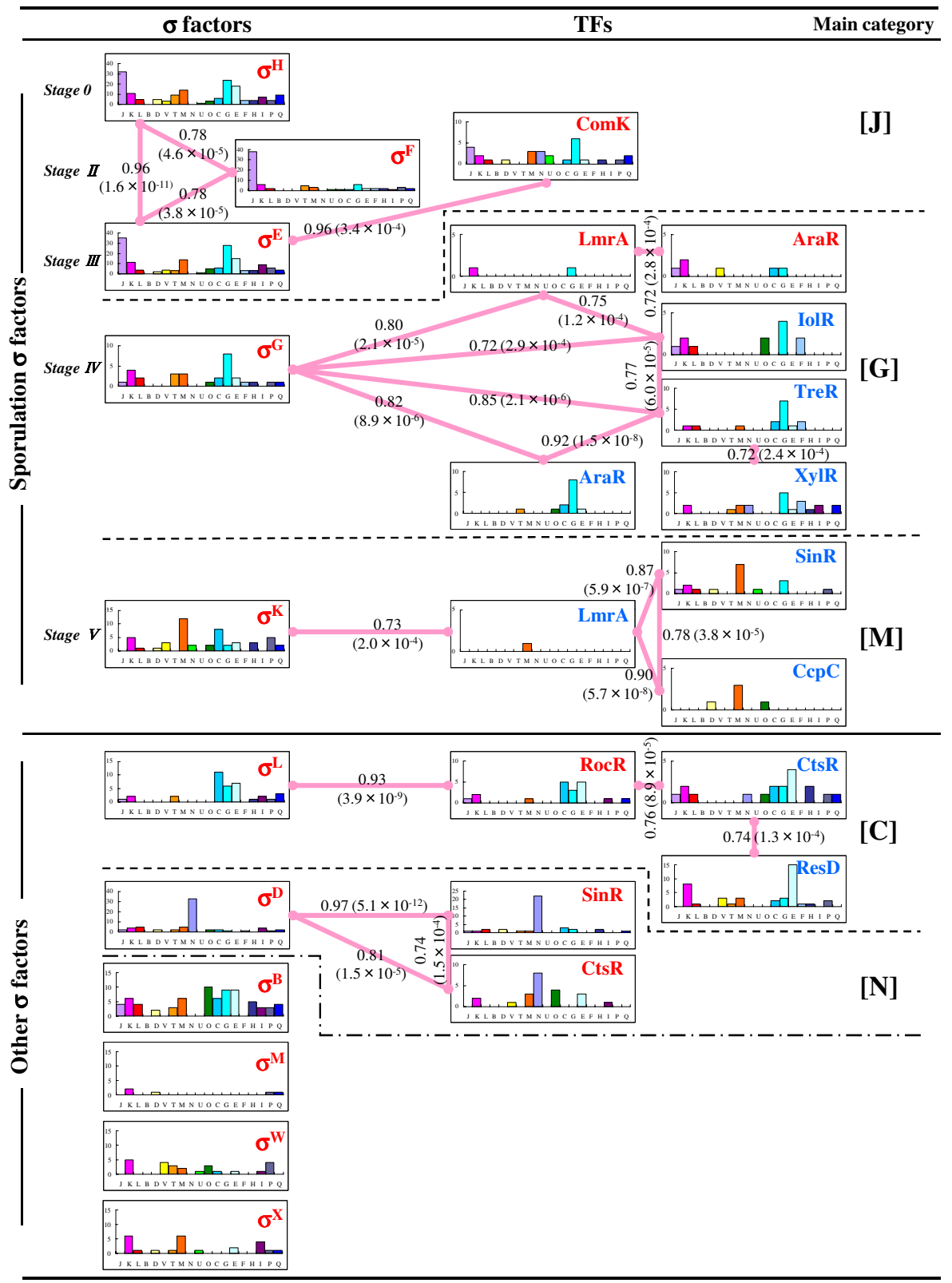

\section{Figure 4}

Clustering of TFs based on the functional similarity of genes composing the TUs. For clustering of TFs, Pearson correlation coefficients among TFs were calculated using the frequencies of genes belonging to each of the COG categories. We regarded groups of TFs that have correlation coefficients above 0.70 (solid lines) as the clusters. Pearson correlation coefficients and P-values in the parentheses are represented on the solid lines. The main category represents the COG category into which the most genes regulated by each of the TFs in the clusters are classified. One-letter abbreviations use used for the functional categories: J, translation, ribosomal structure and biogenesis; K, transcription; L, DNA replication, recombination and repair; $\mathrm{D}$, cell division and chromosome partitioning; $\mathrm{O}$, posttranslational modification and protein turnover, chaperones; $\mathrm{M}$, cell envelope biogenesis and outer membrane; $\mathrm{N}$, cell motility and secretion; $\mathrm{P}$, inorganic ion transport and metabolism; $\mathrm{T}$, signal transduction mechanisms; $\mathrm{C}$, energy production and conversion; $\mathrm{G}$, carbohydrate transport and metabolism; $\mathrm{E}$, amino acid transport and metabolism; F, nucleotide transport and metabolism; H, coenzyme metabolism; I, lipid metabolism; Q, secondary metabolite biosynthesis, transport and catabolism. All data were identified under PWM with I\% threshold and FDR control. 

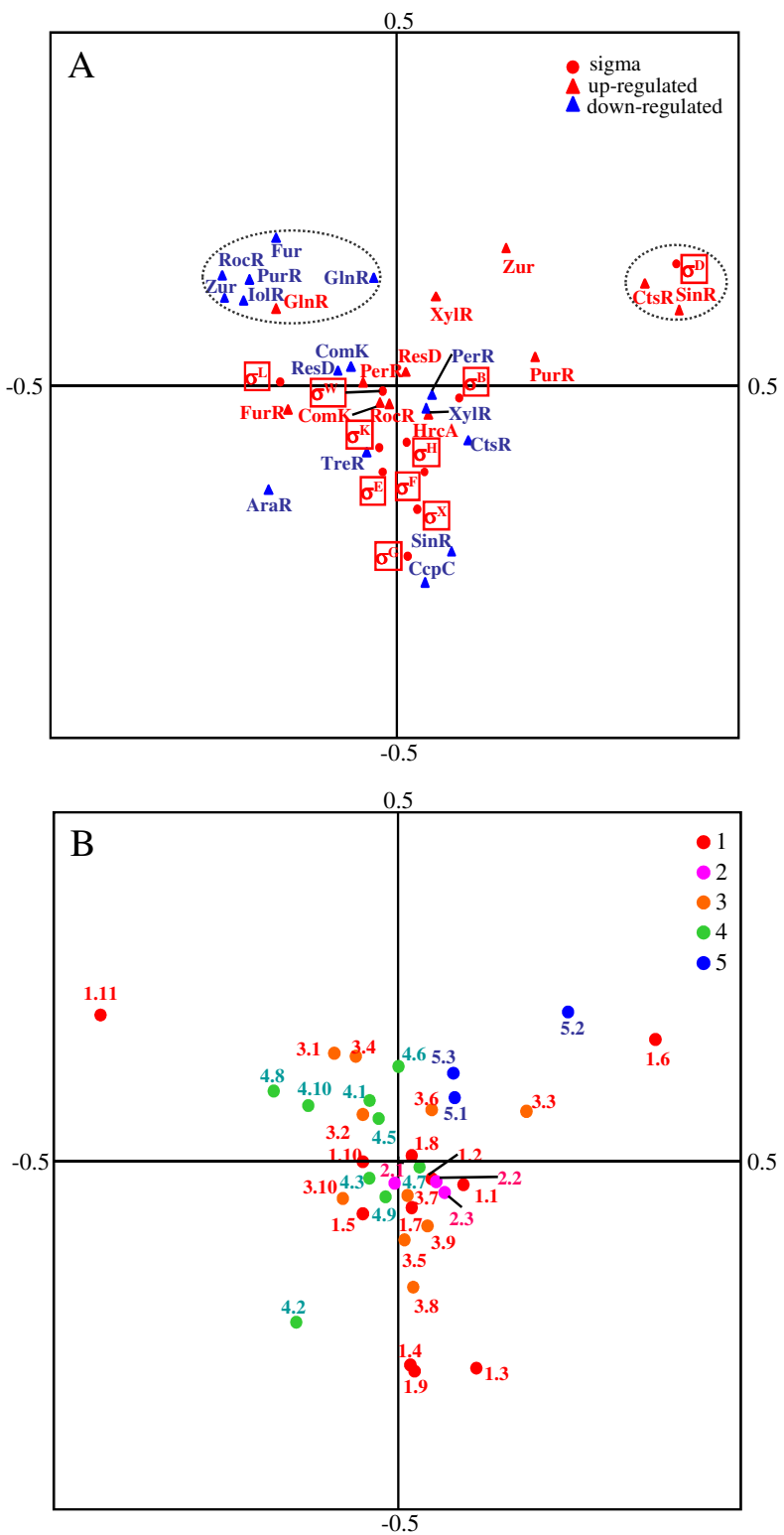

\section{Figure 5}

Score plots for PCA of TFs and functional categories. (A) Score plot based on frequencies of TF functions. (B) Score plot based on frequencies of functional classification of the $B$. subtilis protein-encoding genes. Abbreviations for the functional categories: I, cell envelope and cellular processes [I.I, adaptation to atypical conditions; I.2, cell division; I.3, cell wall; I.4, germination; I.5, membrane bioenergetics (electron transport chain and ATP synthase); I.6, motility and chemotaxis; I.7, protein secretion; I.8, sensors (signal transduction); I.9, sporulation; I.I0, transformation/competence; I.II, transport/binding proteins and lipoproteins]; 2, cytochromes [2. I, cytoplasmic; 2.2, membrane-bound; 2.3, other cytochromes]; 3, information pathway [3.I, competence regulatory; 3.2, detoxification; 3.3, DNA packaging and segregation; 3.4, DNA replication; 3.5, DNA restriction/modification, repair and recombination; 3.6, protein folding; 3.7, protein modification; 3.8, protein synthesis; 3.9, RNA modification; 3.I0, RNA synthesis]; 4, intermediary metabolism [4. I, antibiotic production; 4.2, carbohydrates and related molecules; 4.3, degradation; 4.4, general function prediction; 4.5 , metabolism of amino acids and related molecules; 4.6 , metabolism of coenzymes and prosthetic groups; 4.7, metabolism of lipids; 4.8, metabolism of nucleotides and nucleic acids ; 4.9, metabolism of phosphate; 4.10, metabolism of sulfur]; 5 , other functions [5.I, antibiotic production; 5.2, phage-related functions; 5.3, transposon and insertion elements]. 
port, metabolism, and biosynthetic pathways [45]. In this cluster, RocR and GlnR relate to controlling nitrogen sources: RocR controls arginine catabolism [46] and the arginase pathway in which arginine is converted to glutamate [47], while GlnR regulates responses to nitrogen availability, such as nitrogen metabolism [48] and assimilation [49].

Based on these previous studies, this result shows that we can cluster together homeostatic regulation TFs (Fig. 5A). Moreover, $\sigma$ factors that regulate sporulation $(\sigma \mathrm{E}, \sigma \mathrm{F}, \sigma \mathrm{G}$, $\sigma \mathrm{H}, \sigma \mathrm{K}$ ) tend to exist near the $\mathrm{y}$-axis in the region of lower first-principal component (PC1) values with negative PC2 values, and TreR, SinR, and CcpC are also plotted near the $\sigma$ factors (Fig. 5A). TreR regulates trehalose as the sole carbon and energy source of $B$. subtilis during spore outgrowth [50], while SinR controls regulatory genes involved in the early stages of sporulation [51]. Thus, sporulation-related TFs tend to have lower PC1 values and negative PC2 values, which may be evidence that category [1.9; sporulation] and [1.4; germination] are plotted in the area (Fig. 5B). Therefore, CcpC is known to be a regulator of the tricarboxylic acid cycle genes [52], but may also have a function in regulating sporulation genes.

\section{Conclusion}

This study presented the new approach to TU prediction in the bacterial whole genome using integrated analysis of microarray and DNA sequence data, and we efficiently detected genes composing TUs in B. subtilis genome. The results demonstrate that the combined approach is very useful for identifying unknown TUs in the genome, and also detecting internal operons in the known operons. This methodology should contribute to studies of predicting TU locations in the bacterial genome and estimating roles of TFs.

\section{Methods}

\section{Bacterial strains, medium, growth conditions and RNA extraction}

For expression profile analyses, B. subtilis 168 was grown in 13 different time-series growth conditions: anaerobic growth; competent medium; cold-shock experiments; DSM medium; DGG medium; glucose-limited medium; heat-shock experiment; LB medium; minimum-glucose medium; sodium-shock conditions; phosphate-starvation medium; and SOS stress experiments. For TF deletion mutant analyses, TF deletion mutants were grown at $37^{\circ} \mathrm{C}$ in different medium conditions: LB medium for sigB, $L$, $M, W, X$, araR, ctsR, hrcA, iolR, ImrA, rocR, sinR, xylR deletion mutants; LB medium with trace elements for fur and perR deletion mutants; DSM medium for sigD, E, F, G, $H$, $K$, treR deletion mutants; DSM medium with $2 \%$ Gln and $5 \%$ glucose for, respectively, $g \ln R$ and resD deletion mutants; MC medium for the comK deletion mutant;
MGM medium for the $c c p C$ deletion mutant; and MGM with adenine and guanine for the purR deletion mutant. Cells were harvested by centrifugation at $1,000 \mathrm{~g}$ after adding the RNA-protecting Bacteria Reagent (Qiagen), and then stored at $-80^{\circ} \mathrm{C}$. Two independent samplings were performed. RNA was isolated using the RNA protectant, RNeasy Mini and RNase-free DNase kits (Qiagen) according to the manufacturer's instructions and stored at $-80^{\circ} \mathrm{C}$. Genomic contamination was estimated by gel electrophoresis.

\section{Labeling}

For each labeling reaction, a total of $15 \mu \mathrm{g}$ of RNA was used. First-strand cDNA synthesis was primed with $1.2-\mu \mathrm{g}$ random primers (Invitrogen) in nuclease-free water (total volume: $31 \mu \mathrm{l}$ ) by heating at $70^{\circ} \mathrm{C}$ for $10 \mathrm{~min}$ and incubating at $25^{\circ} \mathrm{C}$ for an additional $10 \mathrm{~min}$. Reverse transcription was performed by SuperScript III (Invitrogen) in reverse transcription buffer $[1 \times$ first-strand buffer, $10 \mathrm{mM}$ DTT] in the presence of $5 \mathrm{mM}$ dATP, $5 \mathrm{mM}$ dUTP, $5 \mathrm{mM}$ dCTP, $0.25 \mathrm{mM}$ dTTP, and $0.25 \mathrm{mM}$ AA-dUTP. Three amino-allyl-labeled nucleotides were incorporated into the cDNA. The reactions were incubated at $25^{\circ} \mathrm{C}$ for 10 $\min , 37^{\circ} \mathrm{C}$ for $60 \mathrm{~min}, 42^{\circ} \mathrm{C}$ overnight, and quenched by heating at $70^{\circ} \mathrm{C}$ for $10 \mathrm{~min}$.

The RNA template was hydrolyzed by adding $20 \mu \mathrm{l}$ of $1 \mathrm{~N}$ $\mathrm{NaOH}$ followed by heating at $65^{\circ} \mathrm{C}$ for $30 \mathrm{~min}$. Reactions were neutralized with $20 \mu \mathrm{l}$ of $1 \mathrm{~N} \mathrm{HCl}$. cDNA was purified using a CyScribe GFX Purification Kit (GE Healthcare) according to the manufacturer's directions. NHS ester forms of Cy3 and Cy5 dyes were added to the cDNA solution and incubated for $4 \mathrm{hr}$. Coupling reactions were quenched by the addition of $15 \mu \mathrm{l}$ of $4 \mathrm{M}$ hydroxylamine and incubated at room temperature for $15 \mathrm{~min}$ in the dark. Labeled cDNA was purified using the CyScribe GFX Purification Kit again.

\section{Hybridization and spot detection}

Prehybridization of the array slides was performed for $3 \mathrm{hr}$ in filtered prehybridization solution [25\% formamide, $5 \times$ SSC, $10 \mathrm{mg}$ BSA (fraction V), $0.1 \%$ SDS] at $42^{\circ} \mathrm{C}$. Slides were briefly washed in milliQ water and $80 \%$ ethanol and dried by centrifugation at $1,000 \mathrm{~g}$ for $5 \mathrm{~min}$. Hybridization of the probe was performed using hybridization solution $(25 \%$ formamide, $5 \times$ SSC, $0.1 \%$ SDS, $0.1 \mu$ g poly(A), $1 \times$ Denhardt's solution and 100 pmol Cy3 and Cy5 combined probe). The hybridization solution containing the Cy-dye-labeled cDNA was heated to $95^{\circ} \mathrm{C}$ for $3 \mathrm{~min}$ and hybridization was performed in an Advalytix hybridization machine (ArrayBooster) at $42^{\circ} \mathrm{C}$ for $16 \mathrm{hr}$. After hybridization, the slides were washed and dried by centrifugation at $1,000 \mathrm{~g}$ for $5 \mathrm{~min}$ and then analyzed using a Fuji FLA-8000 scanner and Array Gauge ver.2.0 software (Fuji Film). 


\section{Normalization in microarray experiments}

Gene expression levels are evaluated by scanning the fluorescence intensity for each spot, and there is usually some experimental variation that occurs in every microarray experiment. It is, therefore, important to minimize experimental variation, and although several methods of microarray normalization have been developed [53,54], there are usually some false-positive data arising when analyzing gene expression data collected via microarrays.

Normalization of the logarithmic ratio of expression intensity between target $\left(\mathrm{R}_{\mathrm{i}}\right)$ and control $\left(\mathrm{G}_{\mathrm{i}}\right)$ experiments was carried out based on MA plots [55], which can show the intensity-dependent ratio of raw microarray data using TREBAX [56]. The plots differed in the axes used. The MA plot used $M_{i}\left(\log _{10}\left(R_{i} / G_{i}\right)\right)$ as the y-axis and $A_{i}\left(\log _{10}\right.$ $\sqrt{R_{i} G_{i}}$ ) as the x-axis. By plotting values of $A_{i}$ on the abscissa and $\mathrm{M}_{\mathrm{i}}$ on the ordinate of a coordinate system, it was possible to evaluate the bias error with respect to the average logarithmic intensities. The normalized log ratio $\mathrm{M}_{\mathrm{i}}{ }_{\mathrm{i}}$ was estimated as the difference between $\mathrm{M}_{\mathrm{i}}$ and baseline $\mathrm{M}_{\mathrm{i}}^{\prime}$. Here, using the relation between $\mathrm{M}_{\mathrm{i}}$ and $\mathrm{A}_{\mathrm{i}}\left(\mathrm{M}_{\mathrm{i}}=\right.$ $f\left(\mathrm{~A}_{\mathrm{i}}\right)+\varepsilon_{i}$, where $\varepsilon_{i}$ is the difference between $\mathrm{M}_{\mathrm{i}}$ and $f\left(\mathrm{~A}_{\mathrm{i}}\right)$ for gene i) for the MA plot, the baseline for the $i$ th gene was estimated by $\mathrm{M}_{\mathrm{I}}^{\prime}=f\left(\mathrm{~A}_{\mathrm{i}}\right)$. Genes whose signal intensity was regarded as zero were eliminated from the present analysis. With this methodology, it is assumed that there was no large error due to expression intensity in the majority of the spots.

\section{Prediction of co-expressed gene clusters}

Co-expressed gene clusters were predicted based on expression profiles and genomic locations. The expression profile of the $i$ th position gene is represented by vector $\mathrm{x}_{i^{\prime}}$ consisting of logarithmic ratios for microarray experiments. The algorithm for predicting co-expressed gene clusters is as follows: we selected a series of genes transcribed in the same direction with no intervening gene transcribed in the opposite direction. The genes were denoted $g_{1}, g_{2}, \ldots g_{i}, \ldots, g_{M}$ from their 5 ' to 3 ' termini. Here, $\mathrm{g}_{\mathrm{i}}$ and $\mathrm{g}_{\mathrm{i}+1}(\mathrm{i}=1,2, \ldots, \mathrm{M}-1)$ are adjacent genes on the same DNA strand. First, Pearson correlation coefficients $\left(\mathrm{r}_{\mathrm{st}}\right)$ were estimated for all pairs of vectors $x_{s}$ and $x_{t}(\mathrm{~s}=1,2, \ldots$, $M ; t=1,2, \ldots, M)$. Second, a pair of genes was assigned to a candidate group. Gene $\mathrm{g}_{\mathrm{s}}$ always belonged to group $\mathrm{G}_{\mathrm{s}}(\mathrm{s}$ $=1,2, \ldots, M)$. All the genes $g_{s+1}, g_{s+2}, \ldots, g_{s+T s}$, whose correlations $r_{s(s+1)}, r_{s(s+2)}, \ldots, r_{s T s}$ were statistically significant in a t-test at the $5 \%$ significance level, were classified into $G_{s}$. In the same manner, all the genes $\mathrm{g}_{\mathrm{s}-1}, \mathrm{~g}_{\mathrm{s}-2}, \ldots, \mathrm{g}_{\mathrm{s}-\mathrm{Us}}$, whose correlations $r_{s(s-1)}, r_{s(s-2)}, \ldots, r_{s u s}$ were statistically significant in a t-test at the $5 \%$ significance level, were also clas- sified into $G_{s}$. Thus, altogether $T_{s}+U_{s}+1$ genes were classified into group $G_{s}$. Finally, all members of group $G_{s}$ $(s=1,2, \ldots, M)$ were compared. We counted the number of groups consisting of identical members among $\mathrm{G}_{\mathrm{s}}(\mathrm{s}=$ $1,2, \ldots, M)$ and selected the group having the highest count as the first co-expressed gene cluster $\mathrm{T}_{1}$. After excluding the $T_{1}$ genes from all the groups ( $g_{1}$ to $\left.g_{M}\right)$, we selected the next-highest identical group as the next coexpressed gene cluster $\mathrm{T}_{2}$. This procedure was carried on until the number of members in $T_{v}$ was zero, or until all positions $\mathrm{j}(\mathrm{j}=1,2, \ldots, \mathrm{M})$ were occupied by genes belonging to $\mathrm{T}_{\mathrm{v}}$.

\section{Identification of promoter sequences by PWM}

DNA sequences recognized by TFs consist of consensus regions. We searched for sequences highly homologous to those known to be recognized by TFs using PWM. First, we prepared datasets of training sequences consisting of experimentally determined promoters from DBTBS [57] and "B. subtilis and Its Closest Relatives: from Genes to Cells" [37], which were aligned on the basis of their consensus regions. PWMs for individual TFs were constructed by the frequencies $F_{A k}, F_{T k}, F_{G k}$, and $F_{C k}$ of the four nucleotides (A, T, G, C) in the $k$ th position, including the consensus regions and the five bases upstream and downstream. We determined the score by multiplying all the frequencies corresponding to a given sequence. Second, the thresholds for the binding sites were determined as follows: 4,000 DNA sequences each comprising 300 nucleotides were generated randomly based on the GC content of B. subtilis. The threshold was defined by the value below which the lowest $95 \%$ of the maximum scores in individual DNA sequences were excluded. Third, within the 300-nucleotide sequence upstream of the protein-coding region, individual TF binding sites were predicted by the maximum PWM score above the threshold because about 95\% of TF binding sites were known to exist in these regions. We chose optimal matrices for each random sequence, and regarded sequences that exceeded the threshold as being regulated by the TF. Therefore, we used these sequences to search for other sequences highly similar to those recognized by TFs. This was done by calculating scores for the partial sequences in the stretch of 300 nucleotides upstream of the protein-coding regions of all B. subtilis genes. Sequences whose scores exceeded a threshold were regarded as TF-binding sites.

\section{Expression analysis of TF deletion mutants of $B$. subtilis}

The normalized fluorescence intensity data were analyzed using a one-sided test to compare the results of the deletion mutant to the control samples, and genes whose expression exceeded the threshold were regarded as TFregulated genes. In lower one-sided tests, we considered genes of decreased expression as being up-regulated by the 
$\mathrm{TF}$, whereas genes of increased expression were considered as down-regulated by the TF in upper one-sided tests.

\section{False discovery rate}

For separating inactive genes from those that were deemed active in the expression analysis of TF deletion mutants, we used the false-discovery rate, an alternative approach to multiple testing [58]. On the assumption that we conducted $m$ multiple tests, the null hypothesis that each gene is differentially expressed is true for $m_{0}$ tests, and the alternative hypothesis is true for $m_{1}\left(=m-m_{0}\right)$. Among the $m_{0}$ null hypotheses, $\mathrm{U}$ hypotheses were declared false-negative and $\mathrm{V}\left(=m_{0}-\mathrm{U}\right)$ hypotheses were declared true-positive. Among the $m_{1}$ alternative hypotheses, T hypotheses were called true-negative and $\mathrm{S}\left(=m_{1}-\mathrm{T}\right)$ hypotheses were called false-positive. $\mathrm{R}(=\mathrm{V}+\mathrm{S})$ is the total number of hypotheses rejected and an observable random variable. The FDR was defined as $\pi_{0}=\mathrm{P}(\mathrm{R}>0) \mathrm{E}$ $(\mathrm{V} / \mathrm{R} \mid \mathrm{R}>0)$, and we thus regarded $\mathrm{R}\left(1-\pi_{0}\right)$ as the number of true active genes.

\section{Authors' contributions}

HK designed and carried out the statistical studies, and drafted the manuscript. JA and NF contributed bioinformatics and statistical studies for sequence analyses. KK and NO designed and conducted the microarray experiments. AA provided bioinformatics support of microarray analyses. KEN and SK contributed substantially to manuscript preparation and editing. NO and SK designed and oversaw the project. All authors read and approved the final manuscript.

\section{Additional material}

\section{Additional file 1}

Supplementary Table 1. Transcription units regulated by the TFs in the Bacillus subtilis genome.

Click here for file

[http://www.biomedcentral.com/content/supplementary/14712164-8-197-S1.pdf]

\section{Additional file 2}

Supplementary Figure 1. The relationship between the number of TUs detected in the present study and the number of genes composing the TUs. Click here for file

[http://www.biomedcentral.com/content/supplementary/14712164-8-197-S2.pdf]

\section{Acknowledgements}

We wish to thank the reviewer for appropriate and kind comments, and lan R. L. Smith of Nara Institute of Science and Technology for his valuable discussions. This work was supported by a Grant-in-Aid for Scientific Research on Priority Areas, "Systems genomics", from the Ministry of Education, Culture, Sports, Science and Technology of Japan.

\section{References}

I. Kunst F, Ogasawara N, Moszer I, Albertini AM, Alloni G, Azevedo V, Bertero MG, Bessieres P, Bolotin A, Borchert S, et al: The complete genome sequence of the gram-positive bacterium Bacillus subtilis. Nature 1997, 390(6657):249-256.

2. Ermolaeva MD, White O, Salzberg SL: Prediction of operons in microbial genomes. Nucleic Acids Res 200I, 29(5): I216-122I.

3. Huerta AM, Salgado H, Thieffry D, Collado-Vides J: RegulonDB: a database on transcriptional regulation in Escherichia coli. Nucleic Acids Res 1998, 26(I):55-59.

4. Salgado H, Moreno-Hagelsieb G, Smith TF, Collado-Vides J: Operons in Escherichia coli : genomic analyses and predictions. Proc Natl Acad Sci USA 2000, 97( I 2):6652-6657.

5. Wang L, Trawick JD, Yamamoto R, Zamudio C: Genome-wide operon prediction in Staphylococcus aureus. Nucleic Acids Res 2004, 32(I 2):3689-3702.

6. Westover BP, Buhler JD, Sonnenburg JL, Gordon Jl: Operon prediction without a training set. Bioinformatics 2005, 21 (7):880-888.

7. Yada T, Nakao M, Totoki $Y$, Nakai K: Modeling and predicting transcriptional units of Escherichia coli genes using hidden Markov models. Bioinformatics 1999, I 5( I 2):987-993.

8. Ogasawara N, Moriya S, Yoshikawa $\mathrm{H}$ : Structure and function of the region of the replication origin of the Bacillus subtilis chromosome. IV. Transcription of the oriC region and expression of DNA gyrase genes and other open reading frames. Nucleic Acids Res 1985, 13(7):2267-2279.

9. Schujman GE, Paoletti L, Grossman AD, de Mendoza D: FapR, a bacterial transcription factor involved in global regulation of membrane lipid biosynthesis. Dev Cell 2003, 4(5):663-672.

10. Moreno-Campuzano S, Janga SC, Perez-Rueda E: Identification and analysis of DNA-binding transcription factors in Bacillus subtilis and other Firmicutes - a genomic approach. BMC genomics 2006, 7: 147.

II. Stormo GD: DNA binding sites: representation and discovery. Bioinformatics 2000, 16(1):16-23.

12. Qiu P, Qin L, Sorrentino RP, Greene JR, Wang L, Partridge NC: Comparative promoter analysis and its application in analysis of PTH-regulated gene expression. J Mol Biol 2003, 326(5): 1327-1336.

13. Heinemeyer T, Wingender E, Reuter I, Hermjakob H, Kel AE, Kel OV, Ignatieva EV, Ananko EA, Podkolodnaya OA, Kolpakov FA, et al: Databases on transcriptional regulation: TRANSFAC, TRRD and COMPEL. Nucleic Acids Res 1998, 26(I):362-367.

14. Kobayashi K, Ogura M, Yamaguchi H, Yoshida K, Ogasawara N, Tanaka T, Fujita $Y$ : Comprehensive DNA microarray analysis of Bacillus subtilis two-component regulatory systems. J Bacteriol 200I, I 83(24):7365-7370.

15. Fujita $Y$, Fujita $T$ : The gluconate operon gnt of Bacillus subtilis encodes its own transcriptional negative regulator. Proc Natl Acad Sci USA 1987, 84(13):4524-4528.

16. Henikoff S, Haughn GW, Calvo JM, Wallace JC: A large family of bacterial activator proteins. Proc Natl Acad Sci USA 1988, 85( I 8):6602-6606.

17. Pawitan Y, Michiels S, Koscielny S, Gusnanto A, Ploner A: False discovery rate, sensitivity and sample size for microarray studies. Bioinformatics 2005, 2 I (13):3017-3024.

18. Gao G, Le D, Huang L, Lu H, Narumi I, Hua Y: Internal promoter characterization and expression of the Deinococcus radiodurans pprl-folP gene cluster. FEMS Microbiol Lett 2006, 257(2): $|95-20|$.

19. Asai K, Takamatsu H, Iwano M, Kodama T, Watabe K, Ogasawara N: The Bacillus subtilis yabQ gene is essential for formation of the spore cortex. Microbiology 200I, I47(Pt 4):919-927.

20. Huang $X$, Helmann JD: Identification of target promoters for the Bacillus subtilis sigma $X$ factor using a consensus-directed search. J Mol Biol 1998, 279(I): I65-173.

21. Levin PA, Losick R: Characterization of a cell division gene from Bacillus subtilis that is required for vegetative and sporulation septum formation. I Bacteriol 1994 I76(5): I 45I-| 459.

22. Hecker M, Schumann W, Volker U: Heat-shock and general stress response in Bacillus subtilis. Mol Microbiol 1996, 19(3):417-428.

23. Wiegeshoff F, Beckering CL, Debarbouille M, Marahiel MA: Sigma L is important for cold shock adaptation of Bacillus subtilis. J Bacteriol 2006, I 88(8):3130-3133. 
24. Marquez-Magana LM, Chamberlin MJ: Characterization of the sigD transcription unit of Bacillus subtilis. J Bacteriol 1994, I 76(8):2427-2434.

25. Horsburgh MJ, Moir A: Sigma M, an ECF RNA polymerase sigma factor of Bacillus subtilis 168 , is essential for growth and survival in high concentrations of salt. Mol Microbiol 1999 32(I):4I-50.

26. Turner MS, Helmann JD: Mutations in multidrug efflux homologs, sugar isomerases, and antimicrobial biosynthesis genes differentially elevate activity of the $\operatorname{sigma}(X)$ and sigma(W) factors in Bacillus subtilis. J Bacteriol 2000, I 82(1 8):5202-5210.

27. Tatusov RL, Koonin EV, Lipman DJ: A genomic perspective on protein families. Science 1997, 278(5338):63I-637.

28. Tovar-Rojo F, Cabrera-Martinez RM, Setlow B, Setlow P: Studies on the mechanism of the osmoresistance of spores of Bacillus subtilis. J Applied Microbiol 2003, 95(I):167-179.

29. Setlow P: Mechanisms for the prevention of damage to DNA in spores of Bacillus species. Annu Rev Microbiol 1995, 49:29-54.

30. Hilbert DW, Piggot PJ: Compartmentalization of gene expression during Bacillus subtilis spore formation. Microbiol Mol Biol Rev 2004, 68(2):234-262.

31. Nakatani Y, Nicholson WL, Neitzke KD, Setlow P, Freese E: SigmaG RNA polymerase controls forespore-specific expression of the glucose dehydrogenase operon in Bacillus subtilis. Nucleic Acids Res 1989, 17(3):999-1017.

32. Magill NG, Cowan AE, Leyva-Vazquez MA, Brown M, Koppel DE, Setlow P: Analysis of the relationship between the decrease in $\mathrm{pH}$ and accumulation of 3-phosphoglyceric acid in developing forespores of Bacillus species. J Bacteriol 1996, I 78(8):2204-2210.

33. Lorca G, Winnen B, Saier MH Jr: Identification of the L-aspartate transporter in Bacillus subtilis. J Bacteriol 2003, I 85(10):3218-3222.

34. Sa-Nogueira I, Mota LJ: Negative regulation of L-arabinose metabolism in Bacillus subtilis : characterization of the araR (araC) gene. J Bacteriol 1997, I79(5): I598-1608.

35. Hoa TT, Tortosa P, Albano M, Dubnau D: Rok (YkuW) regulates genetic competence in Bacillus subtilis by directly repressing comK. Mol Microbiol 2002, 43(I): I5-26.

36. Kearns DB, Chu F, Branda SS, Kolter R, Losick R: A master regulator for biofilm formation by Bacillus subtilis. Mol Microbiol 2005 55(3):739-749.

37. Sonenshein AL, Hoch JA, Losick RM: Bacillus subtilis and Its Closest Relatives: from Genes to Cells. ASM Press 200I.

38. Mirel DB, Chamberlin MJ: The Bacillus subtilis flagellin gene (hag) is transcribed by the sigma 28 form of RNA polymerase. I Bacteriol | 989, I I I(6):3095-3I0I.

39. Derre I, Rapoport G, Devine K, Rose M, Msadek T: ClpE, a novel type of HSP I00 ATPase, is part of the CtsR heat shock regulon of Bacillus subtilis. Mol Microbiol I999, 32(3):58I-593.

40. Msadek T, Dartois V, Kunst F, Herbaud ML, Denizot F, Rapoport G: ClpP of Bacillus subtilis is required for competence development, motility, degradative enzyme synthesis, growth at high temperature and sporulation. Mol Microbiol 1998, 27(5):899-9| 4.

41. Guillen N, Weinrauch Y, Dubnau DA: Cloning and characterization of the regulatory Bacillus subtilis competence genes comA and comB. I Bacteriol I989, I 7 I ( I 0):5354-536I.

42. Gaballa A, Helmann JD: Identification of a zinc-specific metalloregulatory protein, Zur, controlling zinc transport operons in Bacillus subtilis. | Bacteriol 1998, I80(22):58|5-582I.

43. Ollinger J, Song KB, Antelmann H, Hecker M, Helmann JD: Role of the Fur regulon in iron transport in Bacillus subtilis. I Bacteriol 2006, I 88(10):3664-3673.

44. Yoshida KI, Aoyama D, Ishio I, Shibayama T, Fujita Y: Organization and transcription of the myo-inositol operon, iol, of Bacillus subtilis. J Bacteriol 1997, I79(14):459|-4598.

45. Ebbole DJ, Zalkin $\mathrm{H}$ : Bacillus subtilis pur operon expression and regulation. | Bacteriol |989, I7|(4):2|36-2|4|.

46. Gardan R, Rapoport G, Debarbouille M: Expression of the rocDEF operon involved in arginine catabolism in Bacillus subtilis. J Mol Biol 1995, 249(5):843-856.

47. Belitsky BR, Sonenshein AL: An enhancer element located downstream of the major glutamate dehydrogenase gene of Bacillus subtilis. Proc Natl Acad Sci USA 1999, 96( I 8): 10290-I0295.
48. Fisher SH: Regulation of nitrogen metabolism in Bacillus subtilis : vive la difference! Mol Microbiol 1999, 32(2):223-232.

49. Magasanik B: Genetic control of nitrogen assimilation in bacteria. Annu Rev Genet 1982, 16:135-168.

50. Kennett $\mathrm{RH}$, Sueoka $\mathrm{N}$ : Gene expression during outgrowth of Bacillus subtilis spores. The relationship between gene order on the chromosome and temporal sequence of enzyme synthesis. J Mol Biol I97I, 60(I):3 I-44.

5I. Cervin MA, Lewis RJ, Brannigan JA, Spiegelman GB: The Bacillus subtilis regulator $\operatorname{Sin} R$ inhibits spollG promoter transcription in vitro without displacing RNA polymerase. Nucleic Acids Res 1998, 26(16):3806-3812.

52. Hanson RS, Cox DP: Effect of different nutritional conditions on the synthesis of tricarboxylic acid cycle enzymes. J Bacteriol 1967, 93(6): |777-| 787.

53. Quackenbush J: Microarray data normalization and transformation. Nat Genet 2002:496-50I.

54. Yang YH, Dudoit S, Luu P, Lin DM, Peng V, Ngai J, Speed TP: Normalization for CDNA microarray data: a robust composite method addressing single and multiple slide systematic variation. Nucleic Acids Res 2002, 30(4):el5.

55. Dudoit S, Fridlyand J, Speed T: Comparison of discrimination methods for the classification of tumors using gene expression data. J Am Stat Ass 2002, 97(457):77-87.

56. TREBAX [http://kanaya.naist.jp/ skanaya/Web/software/trebax/ trebax2.html]

57. DBTBS [http://dbtbs.hgc.jp/]

58. Benjamini $Y$, Hochberg $Y$ : Controlling the false discovery rate a practical and powerful approach to multiple testing. J Roy Stat Soc B Met 1995, 57(1):289-300.

Publish with BioMed Central and every scientist can read your work free of charge

"BioMed Central will be the most significant development for disseminating the results of biomedical research in our lifetime. "

Sir Paul Nurse, Cancer Research UK

Your research papers will be:

- available free of charge to the entire biomedical community

- peer reviewed and published immediately upon acceptance

- cited in PubMed and archived on PubMed Central

- yours - you keep the copyright
BioMedcentral 